\title{
The role of fluorescence methods in environmental monitoring of cyanobacteria in aquatic systems
}

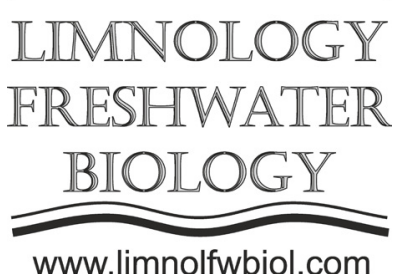

\author{
Grigoryeva N.Y. ${ }^{*}$, Snarskaya D.D. ${ }^{2}$ \\ ${ }^{1}$ Saint-Petersburg Research Center for Ecological Safety, Russian Academy of Sciences, Korpusnaya Str., 18, Saint-Petersburg, 197110, \\ Russia \\ ${ }^{2}$ Saint-Petersburg State University, Botanicheskaya Str. 17A, Saint-Petersburg, 198504, Russia
}

\begin{abstract}
In this article the main advantages of fluorescence spectroscopy in the environmental monitoring of cyanobacteria are pointed out. Several widely used environmental monitoring techniques are discussed in terms of fluorescence spectroscopy data application therein. Two illustrations are given to demonstrate the role of cyanobacterial self-fluorescence spectra study.
\end{abstract}

Keywords: fluorescence spectroscopy, cyanobacteria, environmental monitoring, remote sensing

It is well-known that the self-fluorescence of the photosynthetic apparatus is a powerful tool for investigation of living cyanobacterial communities (Grigoryeva et al., 2018; Grigoryeva, 2019). Such important problems of the environmental monitoring as biological diversity study and cyanobacterial bloom control can be easily solved by means of fluorescence spectroscopy data analysis. Study of the developmental dynamics of cyanobacterial communities by means of non-destructive fluorescence methods gives a unique opportunity of the on-line monitoring and control over toxic cyanobacterial blooms. Moreover, since the physiological state of cyanobacterial cells is closely related to the operation and activity of photosynthetic system, any variations in spectroscopic properties of probed cyanobacterial species indicate the changes in water environment. For example, high concentrations of such environmental pollutants as heavy metals, hormone-like xenobiotics, pesticides, herbicides, polycyclic aromatic hydrocarbons, organic chlorine compounds, etc. can be identified via changes in selffluorescence spectra of cyanobacteria. Finally, it should be noted, that such innovative monitoring methods as biosensing, active biomonitoring and remote sensing cannot be successfully carried out without adjustments taking into account data on self-fluorescence spectra of cyanobacterial species under consideration (Férard and Blaise, 2013).

To demonstrate the usefulness of fluorescence spectroscopy in determination of environmental pollutant action and in cyanobacterial species differentiation two cases are presented in Fig. 1.

Fig. 1A represents changes in self-fluorescence spectra of Synechocystis aquatilis treated by $2 \mathrm{mg} / \mathrm{L}$ copper sulfate pentahydrate. So fast response of photosynthetic apparatus is due to pollutant exposure mechanism. Ions of heavy metals, freely penetrating through cell membrane, directly block light harvesting complex and arrest electron transport in photosystem II reaction center (Lou et al., 2019). Different pollutants induce different changes in spectroscopic properties of photosynthetic apparatus of cyanobacteria, thus the fluorescence methods allow not only to detect the presence of pollutant, but also make some assumptions about the nature of how it affects cyanobacteria (Khishamuddin et al., 2018).

In several works (Yentsch and Phinney, 1985; Grigoryeva, 2019) the possibility of cyanobacterial species discrimination according to their in vivo single-cell fluorescence spectra was shown and a novel technique for ataxonomic on-line analysis was presented. Moreover, several works reported about the possibility of selective determination of cyanobacteria concentration retrieval from hyperspectral data obtained from ground-based, airborne, or satellite sensors by taking advantage of characteristic fluorescent spectral features (Kutser, 2009; Li et al., 2010). For this purpose the investigation of bulk fluorescence spectra of different cyanobacterial species is quite valuable. In Fig. 1B fluorescence spectra of three unicellular cyanobacterial species recorded at excitation wavelength $488 \mathrm{~nm}$ are presented. These species were collected in different countries: Microcystis firma in Turkmenistan, Merismopedia punctata in Cuba, Pinar del Rio, Synechococcus $s p$. in Russia, Saint-Petersburg, and their spectra have characteristic features.

As it was pointed out earlier, fluorescence spectroscopy of cyanobacterial species plays a valuable role in active biomonitoring and remote sensing. Active biomonitoring is a method, when translocation 

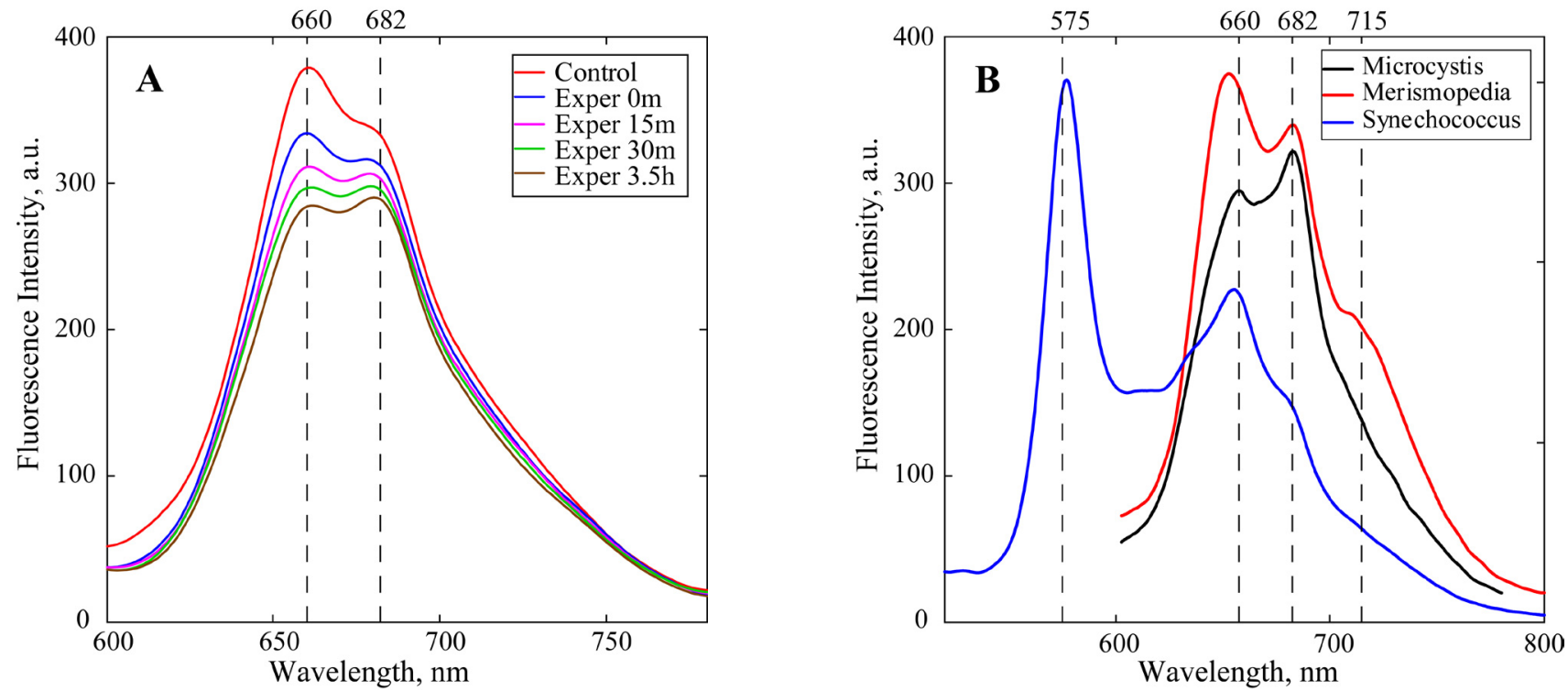

Fig.1. Bulk fluorescence spectra recorded in two different cases: A - series of fluorescence spectra for Synechocystis aquatilis treated by copper sulfate pentahydrate $(2 \mathrm{mg} / \mathrm{L})$. Excitation wavelength $405 \mathrm{~nm}$. Spectra were obtained before and after 0 , 15, 30 minutes and 3.5 hours of pollutant injection. B - characteristic fluorescence spectra of three unicellular cyanobacterial species (Microcystis firma, Merismopedia punctata, Synechococcus sp.), recorded at excitation wavelength $488 \mathrm{~nm}$. Dash lines indicate fluorescence maxima of main photosynthetic pigments: $575 \mathrm{~nm}$ - phycoerythrin, $660 \mathrm{~nm}$ - phycocyanin, $682 \mathrm{~nm}$ - chlorophyll $a$ of photosystem II, $715 \mathrm{~nm}$ - chlorophyll $a$ of photosystem I.

of organisms from one place to another, is followed by quantifying their biochemical, physiological, and organismal responses for the purpose of water quality monitoring (Férard and Blaise, 2013). The main sense of the active biomonitoring is that transplanted organisms (for example, cyanobacteria), collected from unstressed populations, can respond to ambient environmental conditions of polluted sites at an earlier stage and to a greater degree than resident species. In such investigation the ability of fluorescence spectroscopy to fast, cheap and sensitive record of changes in physiological state of cyanobacteria is irreplaceable. As it was shown in Fig. 1A, the photosynthetic apparatus of cyanobacteria responds to the action of heavy metal ions in few minutes. Moreover, to make this procedure faster and automatically processed the measurements may be performed at only one or two spectral points.

Fluorescence spectroscopy data are widely used in detection and quantification of potentially toxic cyanobacteria via remote sensing (Kutser, 2009; Li et al., 2010). This technique is used in ecosystem studies, water quality control and operational monitoring of cyanobacterial occurrence, distribution, and spatial and temporal dynamics in water bodies. For remote sensing it is very important that the presence of cyanobacteria and their photopigments influence "reflectance", which is the ratio of radiation leaving the water (reflected) to the total amount of radiation incident on the water. Thus the knowledge about different fluorescent spectral properties of different cyanobacterial species is needed (see Fig. 1B). There are several techniques to extract the information about different species and their abundance from hyperspectral reflectance, but without spectroscopic data (fluorescence and absorbtion) about these species it is impossible.

\section{Acknowledgments}

Cyanobacterial strains were provided by CALU Collection of the Core Facility Center "Centre for Culture Collection of Microorganisms", Science Park of St.-Petersburg State University. All studies were carried out in "Center for Molecular and Cell Technologies", Science Park of St.-Petersburg State University.

\section{References}

Férard J.F., Blaise C. 2013. Encyclopedia of aquatic ecotoxicology. Dordrecht: Springer. DOI: 10.1007/ 978-94-007-5704-2

Grigoryeva N.Yu., Chistyakova L.V., Liss A.A. 2018. Spectroscopic techniques for estimation of physiological state of blue-green algae after weak external action. Oceanology 58: 896-904. DOI: 10.1134/S0001437018060061

Grigoryeva N. 2019. Self-fluorescence of photosynthetic system - a powerful tool for investigation of microalgal biological diversity. In: M. Vítová (Ed.), Microalgae - from physiology to application. London, pp. 1-22. DOI: 10.5772/ intechopen. 88785

Khishamuddin N.A., Shing W.L., Kin C.M. et al. 2018. Fluorometric response of photosynthetic microorganism consortium as potential bioindicator for heavy metals detection in water. Environment Asia 11: 80-86. DOI: 10.14456/ea.2018.6

Kutser T. 2009. Passive optical remote sensing of cyanobacteria and other intense phytoplankton blooms in coastal and inland waters. International Journal of Remote Sensing 30: 4401-4425. DOI: 10.1080/01431160802562305

Li L., Sengpiel R.E., Pascual D.L. et al. 2010. Using hyperspectral remote sensing to estimate chlorophyll-a and phycocyanin in a mesotrophic reservoir. International Journal of Remote Sensing 31: 4147-4162. DOI: 10.1080/01431161003789549

Lou W., Wolf B.M., Blankenship R.E. et al. 2019. $\mathrm{Cu}^{+}$contributes to the orange carotenoid protein-related 
phycobilisome fluorescence quenching and photoprotection in Cyanobacteria. Biochemistry 58: 3109-3115. DOI: 10.1021/ acs.biochem.9b00409

Yentsch C.S., Phinney D.A. 1985. Spectral fluorescence: an ataxonomic tool for studying the structure of phytoplankton populations. Journal of Plankton Research 7: 617-632. DOI: 10.1093/plankt/7.5.617 